\title{
EMPIRICALLY DETERMINED RESPONSE MATRICES FOR ON-LINE ORBIT AND ENERGY CORRECTION AT THOMAS JEFFERSON NATIONAL ACCELERATOR FACILITY*
}

\author{
A. Hofler, D. Bryan ${ }^{\dagger}$, L. Harwood, M. Joyce, V. Lebedev ${ }^{\ddagger}$, TJNAF, Newport News, VA 23606, \\ USA
}

\begin{abstract}
Thomas Jefferson National Accelerator Facility (Jefferson Lab) uses digital feedback loops (less than 1 $\mathrm{Hz}$ update rate) to correct drifts in the Continuous Electron Beam Accelerator Facility's (CEBAF) electron beam orbit and energy. Previous incarnations of these loops used response matrices that were computed by a numerical model of the machine. Jefferson Lab is transitioning this feedback system to use empirically determined response matrices whereby the software introduces small orbit or energy deviations using the loop's actuators and measures the system response with the loop's sensors. This method is in routine use for orbit correction. This paper will describe the problem that both software lock systems are designed to address. A brief discussion of the design and operational experience of the numerical model based orbit and energy lock software system will follow. Next, the empirical orbit lock system will be described. Finally, future plans for an empirical energy lock system will be presented.
\end{abstract}

\section{PROBLEM DESCRIPTION}

The CEBAF electron beam experiences slow drifts in orbit and energy on the time scale of minutes. The magnitude of the orbit drift does not exceed $1 \mathrm{~mm}$ in position and $0.1 \mathrm{mrad}$ in angle. The magnitude of the energy drift is on the order of $0.001 d p / p$ [1]. Because the time scale is relatively large, these problems do not require specially designed controls hardware to detect and correct the drifts. Therefore, they can be addressed using software running on operator workstations communicating with existing beam diagnostic hardware for sensors, namely beam position monitors (BPMs), and standard CEBAF corrector magnet and RF cavity controls for actuators. Device controls at Jefferson Lab are written using the Experimental Physics and Industrial Control System (EPICS) toolkit [2].

CEBAF can simultaneously deliver to its end station users up to three different beam energies from five total possibilities. Recall that CEBAF is a recirculating linac where its two main anti-parallel linac sections are joined

\footnotetext{
*This work was supported by the U.S. Department of Energy Contract Number DE-AC05-84-ER40150.

†Now with Cisco Systems, San Jose, CA, USA.

"Now with Fermi National Accelerator Laboratory, Batavia, IL, USA.
}

head to tail by nine beam transport systems [3]. The five possible beam energies correspond to five beam extraction points in the beam transport system downstream of the second of CEBAF's two linacs. This implies that in delivering a subset of the available beam energies, it is possible that portions of the beam transport system are unused. For example, if CEBAF is delivering three lower energy beams to end station experiments, the beam transport recirculation arcs associated with the two higher energies are idle. This flexibility in machine configuration means the lock problem needs to be segmented accordingly. It also implies that the corrections of the individual locks have to be applied in such a way as to prevent the locks from fighting each other since the effect of one lock is seen in the sensors of downstream locks.

The position and angle of the beam orbit can be corrected in both the horizontal and vertical planes using four BPM readbacks and four corrector magnets (two sets of two for each plane). In this case, the natural choice for segmentation falls at the beginning of each of CEBAF's beam transport sections that are delineated by points of separation or combination. To this end, CEBAF needs fourteen individual orbit locks for the injection chicane, nine recirculation arcs, and four transport channels.

The energy for each of the CEBAF's linacs can be corrected using the BPM readbacks in the beam transport arc downstream of the linac and four RF cavity voltages at the end of the linac. In addition to the two linacs mentioned, CEBAF has a pre-accelerator linac followed by a beam transport injection chicane that also requires feedback control. In all three cases, the downstream arc is used as a spectrometer to determine the relative beam energy. This method can be applied individually to each linac with the understanding that cross talk between the locks is an issue.

\section{NUMERICAL MODEL BASED ORBIT AND ENERGY LOCK SOFTWARE SYSTEM}

The slow feedback system that uses response matrices from a numerical model of the machine to correct the drifts described has been in use by Accelerator Operations personnel for over four years. This system is used to control the orbit in CEBAF's injection chicane, recirculation arcs, and transport channels and to control the energy for its two main linacs and pre-accelerator linac. It is referred to as the model based lock software, and its design is based on a philosophy that favors the use 
of multiple specialized interdependent software servers [4]. While this design approach allows for parallel development of the individual servers, it ultimately results in a system that requires expert knowledge of the whole system to adequately maintain and support its use.

The model based lock software consists of two parts: the lock engine (server) and the graphical user interface (GUI). The GUI is written using the Tcl/Tk scripting language [5,6] with the $\mathrm{Tcl} / \mathrm{Cdev}$ extension [7]. The server software is written in $\mathrm{C}++$ using the Control Device (CDEV) interface layer [8] to communicate with other CDEV based processes and the EPICS control system. The supporting CDEV based processes include the numerical beam optics model server [9], a BPM readback server to timestamp and distill relevant information for the lock software, and a lock configuration database server to store the lock configuration information. The interplay of these servers makes diagnosing problems with this system difficult.

Reliance on the model server has also proven to be a disadvantage. If a model based lock fails to control the orbit or energy due to some subtle change in the running conditions of the machine, the change has to be identified and either integrated into the model server data or manually corrected in the running machine before the system can respond properly. Either solution is time consuming and adversely affects beam delivery to the end station experiments.

\section{EMPIRICAL ORBIT LOCK SOFTWARE SYSTEM}

The empirical orbit lock software system is considerably simpler in design and does not use any supporting servers [10]. It has been in use by Accelerator Operations personnel for over a year without significant problems. This system is used to control the injection chicane and recirculation arcs and for eight months to control the transport channels. The single server system is written in $\mathrm{C}++$ using CDEV to communicate with the EPICS control system and has a Tcl/Tk and Tcl/Cdev based GUI.

The system's overall streamlined design greatly benefits from the lessons learned with the model based lock system. The system uses one server to perform all the work including data acquisition, data verification, feedback correction calculation, and response matrix determination. Reliability issues related to the BPM server have led to the creation of a composite readback in the low level EPICS BPM software to provide to the locks position and associated RMS information as well as relevant hardware status indicators. This development eliminates the need for the BPM server. Using the sensors and actuators of the lock to derive the response matrix for the optics in the machine obviates the need for the model server. This also allows the orbit lock system to adapt quickly to changes in the running machine.

\subsection{Basic Feedback Control Assumptions}

This system makes the standard system theory assumption that the plant to be controlled is linear or can be adequately represented by a linearized system. Since CEBAF's beam optics to first order are linear, this assumption is valid. The advantage of a linear system, from a controls standpoint, is that a stable solution exists if the eigenvalues of the plant transfer function have negative real parts placing them in the strict left half of the complex s-plane. For optics systems, utilization of quadrupole magnets to define the beam envelope and a judicious choice of corrector magnets to use for correction can minimize the possibility of operating in an unstable region of the state variable phase space. Additionally, the feedback system can be designed around a desired operating set point so the control loop can operate in perturbation mode where only small deviations from the desired set point are allowed or assumed.

\subsection{Lock Calibration}

Lock calibration is the process that allows the system to empirically determine the response matrix. It is central to the success of this system. During a calibration sequence, the software systematically changes each corrector individually to produce a $75-\mu \mathrm{rad}$ kick in the beam orbit followed by a $-75-\mu$ rad kick. The software holds the corrector at each calculated set point for five seconds to allow the BPM readbacks to settle to steady state values. The result of each kick is measured by the BPMs. The error signals, differences between the measured positions and the desired positions (typically $0.0 \mathrm{~mm}$ for each BPM), are collected into a matrix that is then inverted using a Singular Value Decomposition (SVD) algorithm [11]. This inverted matrix is applied to subsequent error signals to calculate the incremental angular kick sizes to apply through the correctors. The angular kicks are converted to magnet strengths, and to minimize cross talk between orbit locks working on adjacent portions of the machine, only a fraction of the calculated correction is written to the EPICS corrector magnet controls. The multiplier applied to the calculated correction to obtain the actual kick is called the fudge factor.

One goal of the empirical orbit lock system is for the software to alert Accelerator Operations personnel when the response matrix for a particular lock is not valid for the present machine optics configuration. To achieve this the lock software examines the focal lengths of neighboring quadrupole magnets in the region of the orbit lock for changes since the last calibration. The algorithm takes advantage of the fact that CEBAF's optics scale linearly with energy. At the time a calibration is performed, the software records the magnet strength set points for the arc bus magnet string, an indication of relative beam energy at the time of the calibration, and the magnet strengths for the quadrupole magnets. As long as the quadrupole magnets are scaled proportionally relative to the arc bus set point, the calibration is valid. The software checks that the present ratios of the individual 
quadrupole strengths to arc bus strength agree with those for the data from the last calibration to within $0.1 \%$ and notifies the Operations crew if the system needs to be recalibrated. The software also recognizes when a system is uncalibrated and notifies the Operations crew accordingly.

Calibration data can be used to characterize the running machine. A history of the magnet strength data can be used to identify inconsistent optics configurations in the operating machine, and analysis of subsequent calibration matrix data can reveal subtle changes over time in the running machine. For these reasons, the calibration data is stored on disk with a timestamp. In addition to the calibration matrix, the element names of the BPMs and all of the magnets and the magnet strengths of the quadrupole and arc bus string magnets are recorded.

\subsection{Lock Operation}

The system is typically operated in one of two configurations corresponding to two accelerator beam operating modes, machine set up and CW beam delivery. For machine set up with pulsed beam, the locks process once every two seconds, and the fudge factor is one half. For CW beam delivery, the locks process once every five seconds using a fudge factor of one tenth. The period and fudge factor can be configured individually for each lock as well as globally for all locks.

\subsection{Diagnostic Information}

When an orbit lock misbehaves, relevant information to have is the list of actions the software performed before the problem occurred. The server maintains a circular buffer of the last $1000 \mathrm{log}$ messages that can be written to disk on-demand for off-line analysis. During normal lock processing, the data recorded to the circular buffer include the measured and desired BPM position information, the error signal, the calculated angular kick correction to apply, and the associated magnet strength after the fudge factor has been applied. Periodically, the empirically determined matrix is also recorded. Other status information is logged such as magnet and BPM status as well as calibration sequence progress.

\subsection{Operational Experience}

The response from Accelerator Operations personnel has been very positive. This is due primarily to that fact the empirical lock system affords the Operations crews an element of control over the locking process that the model server based system does not. With the empirical orbit lock system if a particular lock fails to control the orbit, the Operations crew can recalibrate the system and force the system to adapt to an unidentified change in the running conditions and quickly return to beam delivery operations. The streamlined design also benefits Accelerator Operations when problems arise requiring software support personnel since there is only one server to check and restart if necessary.

\section{FUTURE PLANS FOR EMPIRICAL ENERGY LOCK SOFTWARE}

The empirical energy lock system will be an extension of the empirical orbit lock software. For the calibration sequence, the individual RF cavity voltages of the last four RF cavities will be modified to introduce a +/-0.0001 $d p / p$ change in energy and the plant response will be measured using the BPMs in the downstream arc. The calibration validation scheme will be the same as the one used for the empirical orbit lock system. To enhance robustness, the system will identify malfunctioning BPMs and reconfigure itself accordingly through the use of an over determined system where the number of BPMs exceeds the number of actuators. Also, the software will analyze a time history of applied corrections in order to identify when the response matrix in use is no longer effective.

\section{CONCLUSION}

Jefferson Lab has successfully developed an orbit lock system that empirically determines its plant model. This has been used to replace the model based orbit locks. An analogous energy lock system is planned and will be developed in the next year.

\section{REFERENCES}

[1] Y.-C. Chao, private communication, Jefferson Lab, May 2001.

[2] EPICS home page, http://www.aps.anl.gov/epics/.

[3] C.W. Leeman, D.R. Douglas, and G.A. Krafft, "The Continuous Electron Beam Accelerator Facility: CEBAF at the Jefferson Laboratory," Annual Reviews of Nuclear and Particle Science, to be published.

[4] J. van Zeijts, S. Witherspoon, and W.A. Watson, "Design and Implementation of a Slow Orbit Control Package at Thomas Jefferson National Accelerator Facility," PAC'97, Vancouver, B.C., Canada, May 1997.

[5] J.K. Ousterhout, Tcl and the Tk Toolkit, AddisonWesley Professional Computing Series (1994).

[6] J. van Zeijts, "Rapid Application Development Using the Tcl/Tk Language," PAC'95, Dallas, TX, USA, May 1995.

[7] J. van Zeijts, Tcl/Cdev home page, http://www.rhichome.bnl.gov/People/johannes/cdevtc lman.html.

[8] CDEV home page, http://www.jlab.org/cdev/.

[9] B.A. Bowling, H. Shoaee, S. Witherspoon, and J. van Zeijts, "The Use of Artemis with High Level Applications," ICALEPCS, Chicago, IL, USA, October-November 1995.

[10] D. Bryan, working papers, Jefferson Lab, May 2000

[11] W.H. Press, S. Teukolsky, W.T. Vetterling, and B.P. Flannery, Numerical Recipes in C: The Art of Scientific Computing, Second Edition, Cambridge University Press (1994). 